\title{
An Efficient Segmentation Technique for Different Medical Image Modalities
}

\author{
Amira A. Mahmoud \\ Depart. of Electronics and Electrical \\ Comm., Faculty of Electronic Eng., \\ Menoufia Uni., Egypt. \\ ameragreeda@yahoo.com \\ El-Sayed El-Rabaie \\ Depart. of Electronics and Electrical \\ Comm., Faculty of Electronic Eng., \\ Menoufia Uni., Egypt.
}

\author{
Walid El-Shafai \\ Depart. of Electronics and Electrical \\ Comm., Faculty of Electronic Eng., \\ Menoufia Uni., Egypt. \\ Osama Zahran \\ Depart. of Electronics and Electrical \\ Comm., Faculty of Electronic Eng., \\ Menoufia Uni., Egypt. \\ Fathi E. Abd El-Samie \\ Depart. of Electronics and Electrical \\ Comm., Faculty of Electronic Eng., \\ Menoufia Uni., Egypt.
}

\author{
Taha E. Taha \\ Depart. of Electronics and Electrical \\ Comm., Faculty of Electronic Eng., \\ Menoufia Uni., Egypt. \\ Adel El-Fishawy \\ Depart. of Electronics and Electrical \\ Comm., Faculty of Electronic Eng., \\ Menoufia Uni., Egypt.
}

\begin{abstract}
In this paper, a study of the segmentation of medical images is presented. The paper provides a solid introduction to image enhancement along with image segmentation fundamentals. Firstly, the local spatial information of the image is enhanced with morphological operations to ensure noise-immunity and image detailprotection. The objective of using morphological operations is to remove the defects in the texture of the image. Secondly, fuzzy c-means (FCM) clustering is used with modification of membership function based only on the spatial neighbors instead of the distance between pixels within local spatial neighbors and cluster centers. The proposed technique is very simple to implement and significantly fast, since it is not necessary to compute the distance between the neighboring pixels and the cluster centers. It is also efficient when dealing with noisy images because of its ability to improve membership partition matrix efficiently. Experimental results performed on different medical image modalities illustrate that the proposed technique can achieve good results, as well as short time and efficient image segmentation.
\end{abstract}

Keywords: Image segmentation; Ultrasonic; X-ray; CT; PET; MR; FCM; Morphological operations; Active contour.

\section{INTRODUCTION}

Various medical practitioners seek to retrieve, diagnose and examine diseases from the images created by medical imaging techniques. The major types of medical scans are Ultrasonic (Us), X-ray, Computed Tomography (CT), Emission Computed Tomography (ECT) and Magnetic Resonance (MR) images. These medical images are composed of clinical data, physiological patterns, soft and hard tissues, benign and malignant tumors. These image modalities differ as they reflect the internal anatomy of the different body organs. Morphology refers to the description of shape and structure of the object in a specific image. It depends on the set theory and rely more on the relative arrangement of pixels instead on their numerical values. The important parameters for structuring elements are shape, size and origin.
The structuring element shape depends on the arrangement of ones and zeros in the matrix. The structuring element acts as a window, over which the interaction takes place. It also helps to characterize the image objects or features. The mechanism of structuring element operation is similar to that of masks used in spatial filtering. A structuring element is moved over the whole image [1]. Image segmentation [2,3,4] is the process of splitting an image into a number of non-overlapping segments (sets of pixels, also known as image objects). The success of the image analysis process depends on the accuracy of segmentation process, but a successful segmentation of an image is generally a difficult problem. During an image preprocessing operation, the input is an image and the output is an enhanced high-quality image. Image segmentation is also an important tool in various medical imaging applications. It simplifies the delineation of boundaries or edges of structures, organs, or other regions of interest. A number of techniques have been proposed for image segmentation. Among these techniques, active contour is the most well-known because of its efficiency and speediness. This paper will focus on medical image segmentation based on morphological operations, FCM, and active contour model.

This paper is organized as follows. Section 2 illustrates the work motivation. Section 3 summarizes the related work in the field of medical image segmentation. Section 4 illustrates the procedure of the proposed segmentation process. The simulation results on Us, X-ray, CT, PET and MR images are given in Section 5. Section 6 handles the effect of different noise types on the segmentation process. Finally, Section 7 presents the conclusions and future work followed by the most relevant references.

\section{A. Motivation}

This paper aims to develop segmentation methods for different medical image modalities. In particular, the main target is the segmentation of tumor region of interest (ROI). The segmentation model uses FCM combined with morphological operations and the active contour model to delineate the ROI, which can be extracted and classified as being benign or malignant in the future work. The motivation of this work is to enhance the patient safety by providing 
greater and more accurate information for medical diagnosis. This paper aims to detect, analyze and solve the important and relevant medical problems.

\section{B. RELATED WORK}

Amira Soudani et al. [5] proposed a model based on active contour model for image segmentation. Their model is based on the combination of an adaptive local term based on the computation of local statistics deduced at each point of the evolved curve and a global term built using the means of the intensity inside and the intensity outside the evolved curve. This model introduces an adaptive energy term by the definition of local regions along the curve that are updated at each iteration of the minimization process according to gradient information.

Wei Xu et al. [6] proposed an ensemble of active contourbased image segmentation to obtain the optimal segmentation ensemble through maximizing the weighted mutual information between the probability distributions of multiple segmentation results. Their results validate that the ensemble of contour-based segmentation is robust to the biased initialization. Haider Ali et al. [7] proposed a level-set based model for both global and interactive or selective segmentation tasks. Their model can deal with the intensity in homogeneity and noise. It also can maintain the same performance on clean and noisy images. It employs a combination of a locally computed denoising constrained surface and a denoising fidelity term to make sure of a fine segmentation of local and global features of the image.

Pradipta Sasmal et al. [8] proposed an automated framework for efficient segmentation of such polyps from endoscopic images. Their method uses principal component pursuit (PCP) for specularity reduction and background subtraction, and then the active contour model is used to localize the polyp regions in each frame. Ali Salih et al. [9] proposed a segmentation technique to study and extract the tumors of a breast image. Their method is based on classic morphology and fuzzy morphology. The proposed methods were tested using the database of MIAS, which contained 322 images. After comparison of the statistical results, it is shown that diagnosis of tumor boundary with fuzzy morphology was highly accurate.

It is noticed that several authors introduced a lot of works on medical image segmentation, where they do not work well on images and edges with low contrast, require more sets, take more computational time, have difficulties when segmenting images affected by changes in the tissue characteristics of data, and are not effective for real time applications.

The main contribution of this paper can be summarized as:

1) It presents an efficient segmentation technique for different medical image modalities.

2) It combines FCM with morphological operations so that the tumor region segmentation is represented by the membership functions of the tissues derived from the minimization of the energy in the FCM.

3) It adds both the global and local spatial information into the membership function to decrease the sensitivity problem to the noise and intensity in homogeneity in the image data.
4) It employs the active contour model to delineate the ROI, which can be extracted.

Simulation results on different image modalities show that the proposed technique can achieve segmentation accuracies of $98.83 \%, 99.71 \%, 99.83 \%, 99.85 \%$ and $99.74 \%$ for Us, X-ray, CT, PET and MR images respectively.

\section{The Proposed Image Segmentation Technique}

The proposed image segmentation technique consists of four stages:

Step 1. Pre-processing and enhancement;

Step 2. Morphological operations and FCM;

Step 3. Active contour segmentation;

Step 4. ROI extraction.

The flowchart of the segmentation process is shown in Fig. 1. All steps will be explained in the following subsections.

\section{Step 1. Pre-processing and Enhancement}

Image pre-processing actually means that the input image is fed to a particular algorithm to obtain a better quality image. Image enhancement is known to be the adjustment of a digital image, so that the results are easier and simpler for analysis and display.

\section{Step 2. Morphological Operations and FCM}

For the FCM algorithm [9-12], the rate of convergence is always decided by the distribution characteristics of the data. The FCM is noise sensitive, because the distribution of the image data is affected by the noise, which leads to two problems.

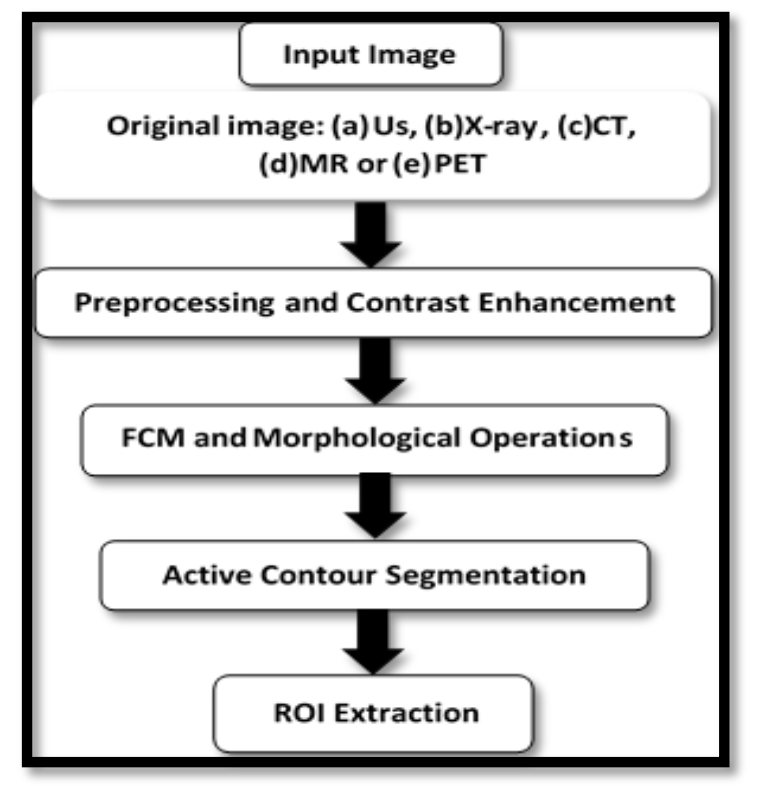

Fig 1. Flowchart of the proposed segmentation technique.

The first is that the output image obtained by FCM algorithm is poor for segmentation; the other is that the number of FCM iterations is larger for an image corrupted by noise $[1,4]$. By introducing local spatial information to the FCM algorithm, it gets immunity to noise and shows superior performance for the segmentation process. The objective function is given by: 


$$
J_{m}=\sum_{l=1}^{q} \sum_{k=1}^{c} \gamma_{l} u_{k l}^{m}\left\|\xi_{l}-v_{k}\right\|^{2}
$$

where $u_{k l}^{m}$ is the FCM of gray value $l$ according to the cluster $k$.

$$
\sum_{l=1}^{q} \sum_{k=1}^{c} \gamma_{l}=N
$$

where $\xi$ is an image obtained by morphological stage, and $\xi_{l}$ is a gray-level, $1 \leq l \leq q, q$ is the number of gray levels in $\xi$. It is less than $N . \xi$ is given as follows:

$$
\xi=R^{c}(I)
$$

where $R^{c}$ denotes the closing morphological operation, and $I$ represents the reference image.

$$
J_{m}=\sum_{l=1}^{q} \sum_{k=1}^{c} \gamma_{l} u_{k l}^{m}\left\|\xi_{l}-v_{k}\right\|^{2}-\lambda\left(\sum_{k=1}^{c} u_{k l}-1\right)
$$

where $\lambda$ is a Lagrange multiplier. Therefore, the problem of the minimization of the objective function is turned into finding the saddle point of the above Lagrange function and taking the derivatives of the Lagrangian $\tilde{J}_{m}$ with respect to the parameters, i.e., $u_{k l}$ and $v_{k}$.

By minimizing the objective function (1), we obtain the corresponding solution as follows:

$$
\begin{gathered}
u_{k l}=\frac{\left\|\xi_{l}-v_{k}\right\|^{-2 /(m-1)}}{\sum_{j=1}^{c}\left\|\xi_{l}-v_{j}\right\|^{-2 /(m-1)}} \\
u_{k}=\frac{\sum_{i=1}^{q} \gamma_{l} u_{k l}^{m} \xi_{l}}{\sum_{l=1}^{q} \gamma_{l} u_{k l}^{m}}
\end{gathered}
$$

According to (5), the matrix of the membership partition $U=\left[u_{k l}\right]^{c \times q}$ is obtained. To obtain a stable $U,(5-6)$ are repeatedly implemented until $\max \left\{U^{(t)}-U^{(t+1)}\right\}<\eta$, where

$\eta$ is a minimal error threshold. Because $u_{k l}^{(t)}$ is a fuzzy membership of gray value $l$ with respect to cluster $k$, a new membership partition matrix $\grave{U}=\left[u_{k l}\right]^{c \times N}$ which corresponds to the original image $I$, is obtained, i.e.

$$
u_{k l}=u_{k l}^{(t)}, \text { if } x_{l}=\xi_{l}
$$

\section{Step 3. Active Contour Segmentation}

Segmentation methods based on the active contour model $[5,6,8]$ outperform many of the traditional image segmentation methods introduced in the literature [13]. Level sets are sophisticated as image segmentation methods that are based upon Partial Differential Equations (PDE) that depend on progressive evaluation of the difference between adjacent neighboring pixels to determine the desired object edges or boundaries.

Level set methods [14-16] are used intensively for segmenting medical images, where they can manage the cavities, concavities, splitting or merging. They depend on a speed function that is calculated from the image gradient. Chan-vese algorithm is based on an energy minimization problem, which can be reformulated in the level set formulation, leading to an easier way to solve the problem. The chan-vese functional is given by:

$$
\begin{aligned}
E\left(c_{1}, c_{2}, \phi\right) & =\lambda_{1} \int_{\Omega}\left(I-c_{1}\right)^{2} H(\phi) d x \\
& +\lambda_{2} \int_{\Omega}\left(I-c_{2}\right)^{2}(1-H(\phi)) d x+\mu \int_{\Omega}|\nabla H(\phi)| d x
\end{aligned}
$$

where $\lambda_{1}, \lambda_{2}>0$ and $\mu \geq 0$ are fixed parameters. The length parameter $\mu$ can be interpreted as a scale parameter, since it determines the relative importance of the length term. The possibility of detecting smaller objects/regions increases with decreasing $\mu$.

The model represents the segmented image with the variables $c_{1}, c_{2}$ and $H(\phi)$, where $H(\phi)$ denotes the Heaviside function of the level set function $\phi$ defined by:

$$
H(\phi(x))= \begin{cases}1 & \phi(x) \geq 0 \\ 0 & \phi(x)<0\end{cases}
$$

The Heaviside function of the level set function, $H(\phi)$, specifies object and back-ground regions in the observed image I, while the last term in (8), $\int_{\Omega}|\nabla H(\phi)|$, expresses the length of the object boundary. On top of that, the scalars $c_{1}$ and $c_{2}$ denote the average gray values of object and background regions indicated by $\phi \geq 0$ and $\phi<0$, respectively.

To segment a given image, the functional (8) needs to be minimized with respect to $c_{1}, c_{2}$, and $\phi$. Keeping $\phi$ fixed, the average gray values $c_{1}$ and $c_{2}$ can be easily estimated by:

$$
\begin{aligned}
& c_{1}=\frac{\int I(x) H(\phi(x)) d x}{\int H(\phi(x)) d x} \\
& c_{2}=\frac{\int I(x)(1-H(\phi(x)) d x}{\int(1-H(\phi(x)) d x}
\end{aligned}
$$

Keeping $c_{1}$ and $c_{2}$ fixed and using the calculus of variations for the functional (8), the gradient descent equation for the evolution of $\phi$ is derived as

$$
\frac{\partial \phi}{\partial t}=\delta(\phi)\left[\mu \nabla \cdot\left(\frac{\nabla}{|\nabla \phi|}\right)-\lambda_{1}\left(I-c_{1}\right)^{2}+\lambda_{2}\left(I-c_{2}\right)^{2}\right]
$$

\section{Simulation Results}

Simulation results were carried out using MATLAB R2019a on a Dell machine, Core I5 processor, 8 Gbytes RAMs and 320 Gbytes hard disk. Simulation results are conducted on five different examples of scanned image (Us, X-ray, CT, ECT and MRI). Images were downloaded from Sirindhorn International Institute of Technology (SIIT) and The Cancer Imaging Archive (TCIA) website $[17,18]$.

To reveal the efficiency of the proposed technique, a sample of each different modality of medical images is tested in the experiments. To assess the segmentation performance of the proposed technique, the accuracy and similarity indices are calculated: the segmentation Accuracy (Acc.), Sensitivity (Sens.), Precision (Pr.), F_Measure (F_M), Matthews Correlation Coefficient (MCC), Dice (D), Jaccard (J) and Specitivity (Spec.) [19]:

$$
\begin{gathered}
\text { Accuracy }=\frac{(T P+T N)}{(F N+F P+T P+T N)} \\
\text { Sensitivity }=\frac{T P}{(T P+F N)} \\
\text { Precision }=\frac{T P}{(T P+F P)} \\
\text { FMeasure }=\frac{2 * T P}{(2 * T P+F P+F N)}
\end{gathered}
$$


MCC

$$
\begin{gathered}
=\frac{(T P * T N-F P * F N)}{\operatorname{sqrt}((T P+F P) *(T P+F N) *(T N+F P) *(T N+} \\
D I C E=\frac{2 * T P}{(2 * T P+F P+F N)} \\
\text { JACCARD }=\frac{D I C E}{(2-D I C E)} \\
\text { Specitivity }=\frac{T N}{(T N+F P)}
\end{gathered}
$$

where $T P$ is the true positive, $F P$ is the false positive, $T N$ is the true negative, $F N$ is the false negative.

Figure (2) illustrates a sample of the different modalities of medical images, (a) is a sample of Us breast image, (b) is a sample of X-ray (mammogram) image, (c) is a sample of CT chest image, (d) is a sample of PET brain image and (e) is a sample of MR brain image. Each of these images has two major areas, a background region and a tumor region. Our aim is to segment and extract the tumor from these images as shown in fig. 3. Figure 3 (a) shows a sample of the reference images, fig. 3 (b) shows the images after pre-processing stage, fig. 3(c) shows the FCM and morphological stage images, fig. 3(d) shows the segmentation results of the ROI produced by active contour model (the tumor is marked by a red contour), and fig. 3(e) shows the resultant ROI images. It is clear that the proposed technique shows an excellent performance for the delineation of the tumor boundaries. The segmentation result can be used in 3D reconstruction of the tumor by computing the volume of the tumor. A physician can make correct diagnosis depending on the variation of the tumor volume. Table (1) shows the segmentation Accuracy (Acc.), Sensitivity (Sens.), Precision (Pr.), F_Measure (F_M), Matthews Correlation Coefficient (MCC), Dice (D), Jaccard (J) and Specitivity (Spec.) numerical results on different image modalities.

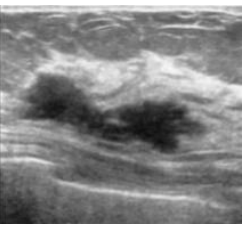

(a)

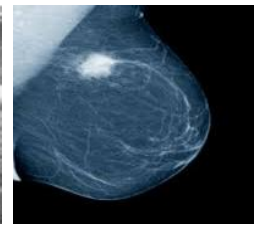

(b)

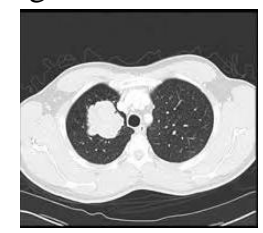

(c)

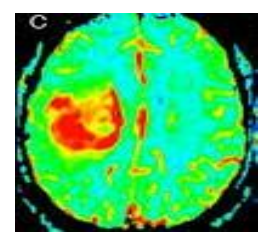

(d)

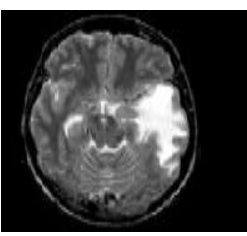

(e)

Fig. 2: A sample of the different modalities of medical images (a) Us image, (b) X-ray image, (c) CT image, (d) PET

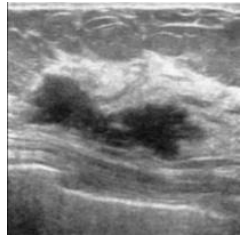

(a)

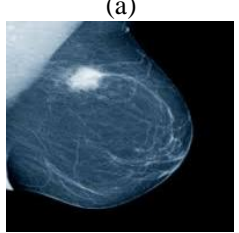

(a)

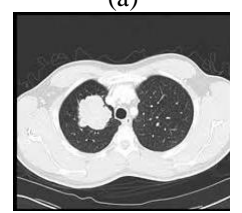

(a)

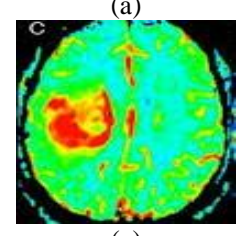

(a)

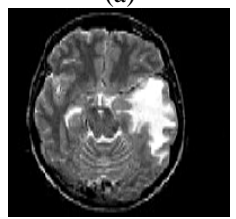

(a)

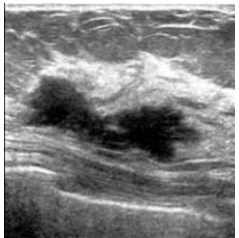

(b)

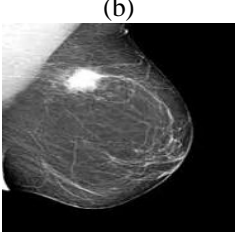

(b)

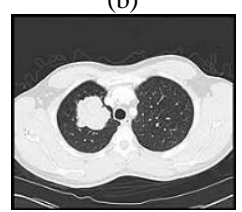

(b)

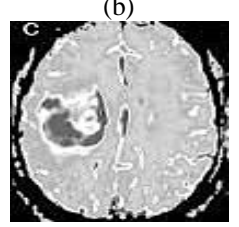

(b)

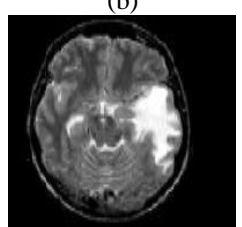

(b)

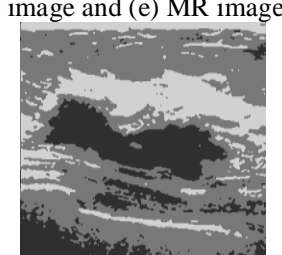

(c)

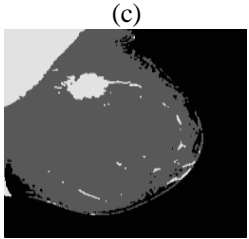

(c)

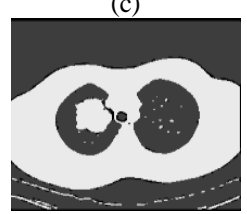

(c)

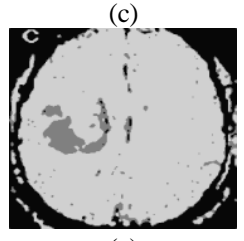

(c)

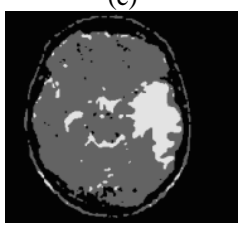

(c)
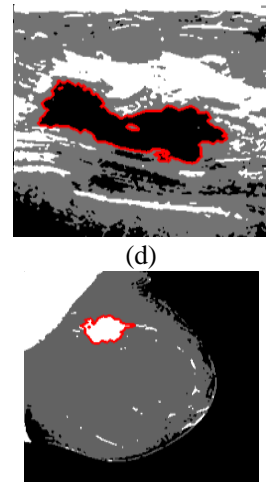

(d)

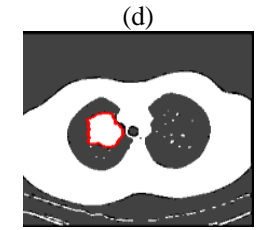

(d)

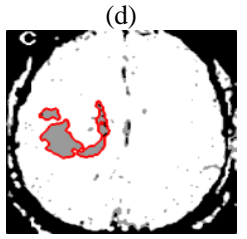

(d)

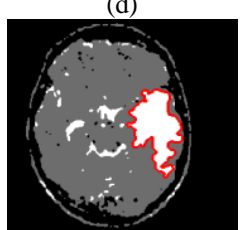

(d)

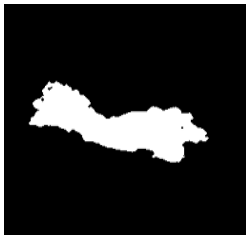

(e)

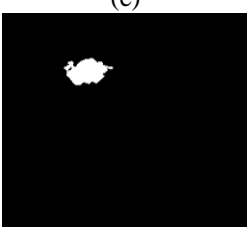

(e)

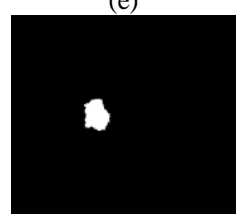

(e)

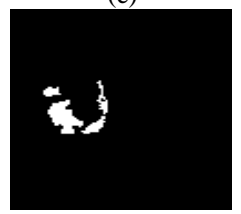

(e)

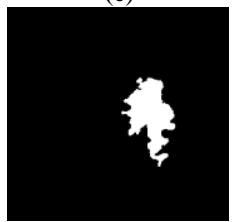

(e)

Fig. 3: (a) The reference image, (b) The image after preprocessing stage, (c) FCM and stage image (d) Segmented image after active contour and (e) The extracted tumor image. 
TABLE 1. PERFORMANCE EVALUATION METRICES OF THE PROPOSED TECHNIQUE ON THE DIFFERENT IMAGE MODALITIES

\begin{tabular}{|c|c|c|c|c|c|c|c|c|}
\hline Image Modality & Acc. & Sens. & F_M & MCC & Pr. & D & J & Spec. \\
\hline Us image & 0.9883 & 0.9077 & 0.9512 & 0.9991 & 0.9460 & 0.9512 & 0.9069 & 0.9999 \\
\hline X-ray image & 0.9971 & 0.9951 & 0.8933 & 0.8104 & 0.8967 & 0.8933 & 0.8072 & 0.9971 \\
\hline CT image & 0.9983 & 0.9211 & 0.9378 & 0.9552 & 0.9371 & 0.9378 & 0.8829 & 0.9994 \\
\hline PET image & 0.9985 & 0.9702 & 0.9693 & 0.9684 & 0.9685 & 0.9693 & 0.9404 & 0.9992 \\
\hline MR image & 0.9974 & 0.9865 & 0.9721 & 0.9581 & 0.9708 & 0.9721 & 0.9457 & 0.9979 \\
\hline
\end{tabular}

\section{NoIse EfFect ON The SEgmentation PROCESS}

It is well known that the distribution characteristics of image data can be described by histogram. If the histogram is uniform, it is difficult to perform a good and fast image segmentation process. On the other hand, it is simple to perform segmentation if the histogram has a number of peaks. Fig. 4 shows an example for each image modality. Speckle noise with 0.05 variance was added to the Us image and Gaussian noise with 0 mean and 0.02 variance was added to the other image modalities. The original image histogram has one or two obvious peaks on contrary the noisy image histogram, which has no obvious peaks.
Fig. 5 (a) shows a sample of the reference images, fig. 5 (b) shows the noisy images, fig. 5 (c) shows the images after pre-processing stage, fig. 5(d) shows the FCM and morphological outputs, fig. 5(e) shows the segmentation results of the ROI produced by active contour model (the tumor is marked by a red contour) and fig. 5(f) shows the resultant ROI images.

Table 2 gives the performance evaluation metrices of the proposed technique on different image modalities in the presence of noise. Table (3) illustrates the execution time (in seconds) of the proposed technique on the different image modalities.

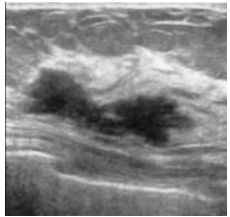

(a)

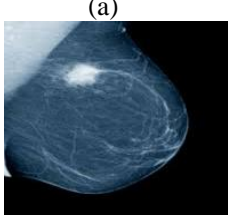

(a)

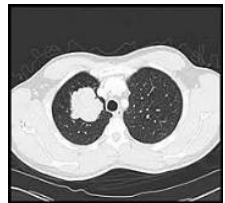

(a)

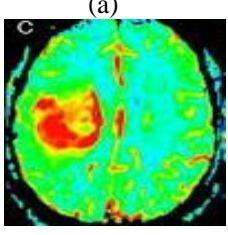

(a)

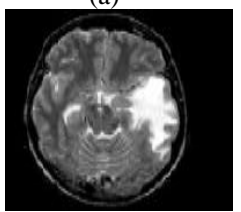

(a)

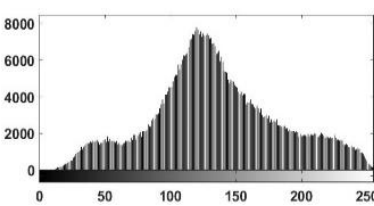

(b)

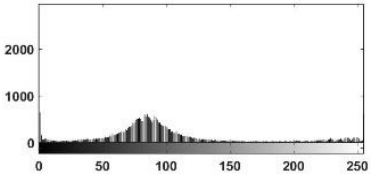

(b)

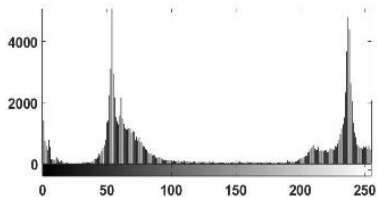

(b)

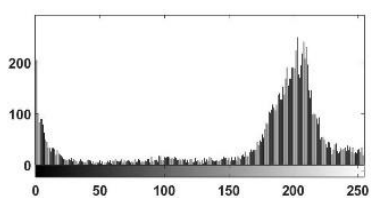

(b)

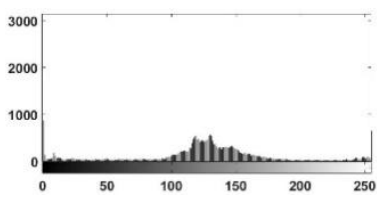

(b)

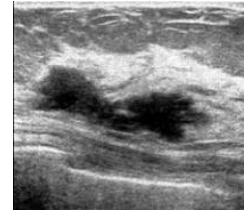

(c)

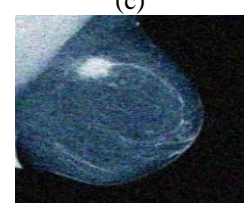

(c)

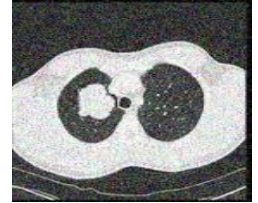

(c)

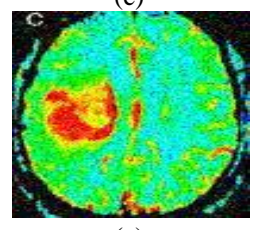

(c)

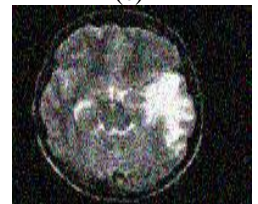

(c)

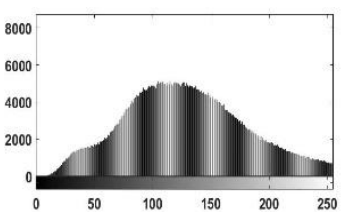

(d)

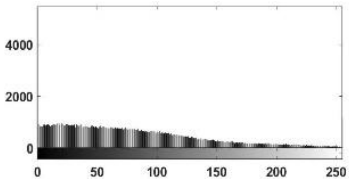

(d)

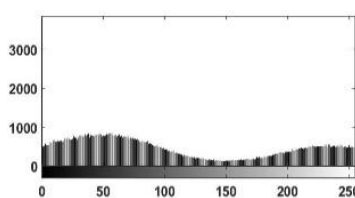

(d)

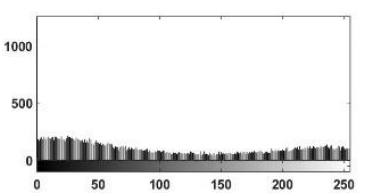

(d)

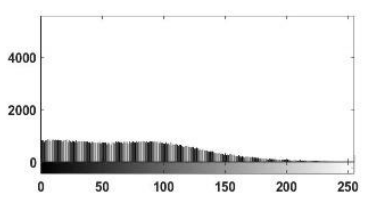

(d)

Fig. 4: Comparison of distributions of data for reference image and noisy image (a) Reference image (b) The reference image histogram, (c) Noisy image and (d) Noisy image histogram 


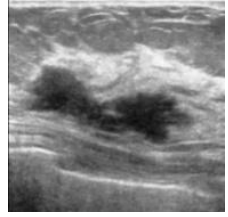

(a)

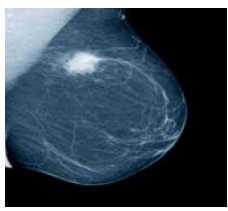

(a)

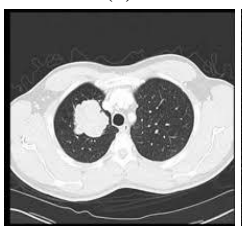

(a)

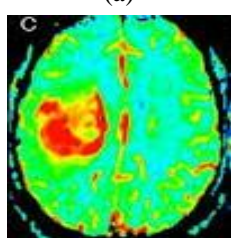

(a)

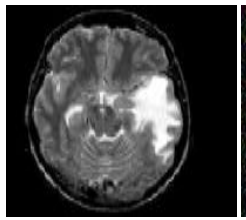

(a)

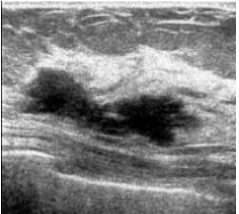

(b)

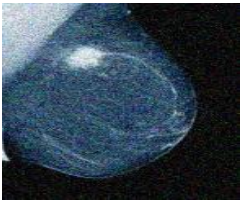

(b)

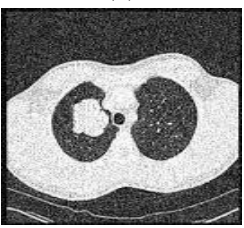

(b)

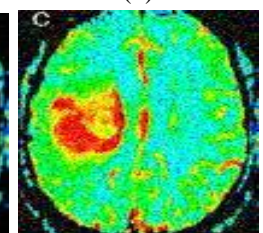

(b)

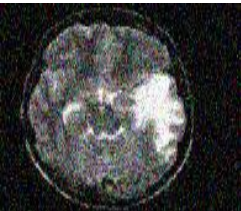

(b)

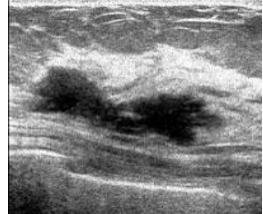

(c)

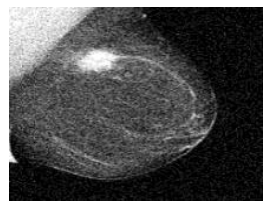

(c)

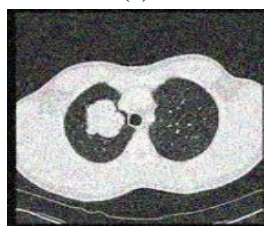

(c)

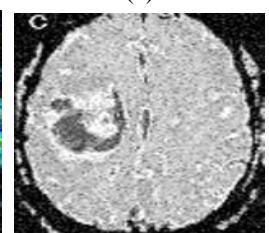

(c)

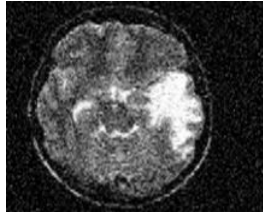

(c)

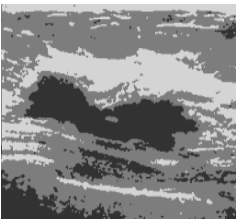

(d)

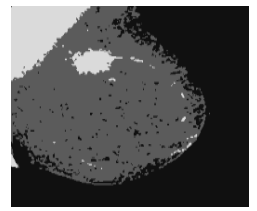

(d)

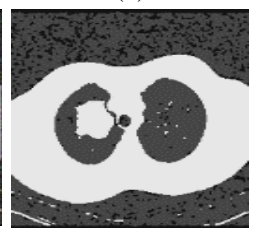

(d)

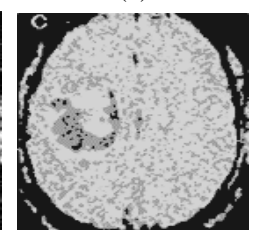

(d)

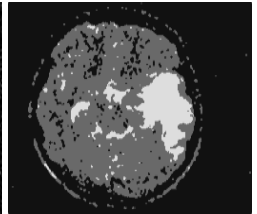

(d)

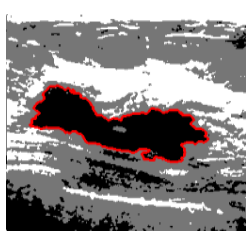

(e)

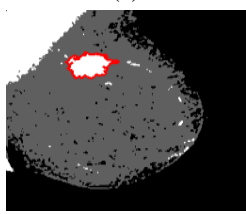

(e)

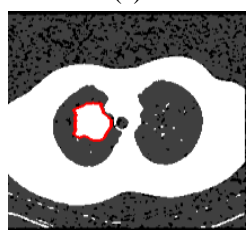

(e)

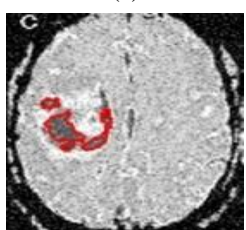

(e)

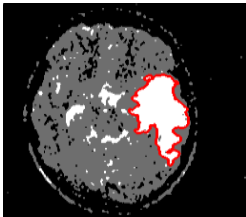

(e)

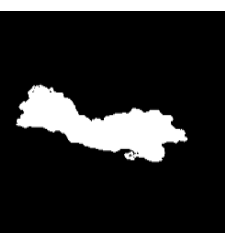

(f)

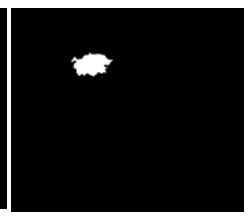

(f)

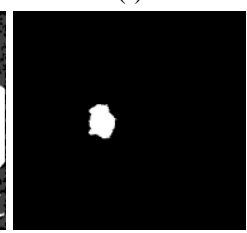

(f)

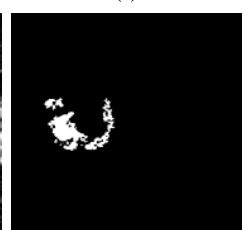

(f)

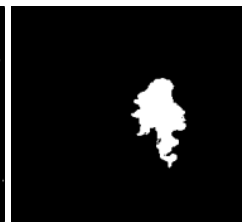

(f)

Fig.5: (a) The reference image, (b) noisy image, (c) image after preprocessing stage, (d) FCM and morphological stage image (e) Segmented image after active contour segmentation and (f) The extracted ROI.

As shown in figures (3), (4) and (5), the technique can segment the image and delineate the tumor boundaries, effectively. Even in the case of segmentation of noisy images, the technique can segment the image and extract the ROI. Table 2 shows good accuracy and similarity results for the undegraded and the degraded images, respectively. It is obvious that combining FCM with the morphological technique can improve the distribution of the image data to apply the segmentation process. Morphological operations can maintain the object boundaries and eliminate noise effect. Based on the composition of erosion and dilation processes, some reconstruction operators with stronger filtering capabilities can be obtained, such as morphological opening and closing reconstructions. The proposed technique has a low complexity because the redundant computation of distance is unnecessary as shown in Table 3 .

TABLE 2. PERFORMANCE EVALUATION METRICS OF THE PROPOSED TECHNIQUE ON THE DIFFERENT IMAGE MODALITIES IN THE PRESENCE OF NOISE.

\begin{tabular}{|c|c|c|c|c|c|c|c|c|}
\hline Image modality & Acc. & Sens. & F_M & MCC & Pr. & D & J & Spec. \\
\hline Us image & 0.9887 & 0.9119 & 0.9527 & 0.9975 & 0.9475 & 0.9527 & 0.9098 & 0.9997 \\
\hline X-ray image & 0.9980 & 0.9335 & 0.9205 & 0.9078 & 0.9195 & 0.9205 & 0.8526 & 0.9988 \\
\hline CT image & 0.9983 & 0.9211 & 0.9378 & 0.9552 & 0.9371 & 0.9378 & 0.8829 & 0.9994 \\
\hline PET image & 0.9921 & 0.7864 & 0.8322 & 0.8837 & 0.8296 & 0.8322 & 0.7126 & 0.9973 \\
\hline MR image & 0.9937 & 0.9644 & 0.9341 & 0.9057 & 0.9313 & 0.9341 & 0.8764 & 0.9951 \\
\hline
\end{tabular}


TABLE 3. THE EXECUTION CPU TIME (IN SECONDS) OF THE PROPOSED TECHNIQUE ON THE DIFFERENT IMAGE MODALITIES

\begin{tabular}{|c|c|c|c|c|c|}
\hline Image Modality & Us & X-ray & CT & PET & MR \\
\hline CPU time (sec) & 2.7 & 1.6 & 1.3 & 1.7 & 1.4 \\
\hline
\end{tabular}

It is easy to obtain good results for image segmentation with the introduction of morphological operations and membership filtering. The illustrated results show that the proposed technique can segment and extract the tumor region successfully even in the presence of different noise types.

\section{CONCLUSIONS AND FUTURE WORK}

In this paper, a significantly fast and robust technique for image segmentation has been proposed to improve the segmentation quality and reduce the influence of image noise. By introducing morphological operations, the local spatial information of the image is utilized to improve the segmentation effect. Because it is possible to suppress noise while preserving the contour of objects, a trade-off has easily been achieved between noise suppression and detail preservation. Moreover, morphological operations are able to provide good reconstructed results for images corrupted by different types of noise. Furthermore, the FCM membership filtering is employed to exploit the local spatial constraints. Results illustrate that the proposed technique gives good segmentation results without tuning parameters for different modalities of medical images. The future work will focus on the presentation of an efficient segmentation stage, as well as, a classification stage for tumors in different multi-modality medical images as a powerful tool for identifying benign ultrasonic breast trumors from malignant ones.

\section{REFERENCES}

[1] Lei, T., Jia, X., Zhang, Y., He, L., Meng, H., \& Nandi, A. K. "Significantly Fast and Robust Fuzzy C-Means Clustering Algorithm Based on Morphological Reconstruction and Membership Filtering", IEEE Transactions on Fuzzy Systems, 2018.

[2] Gang Li, Yi Zhao, Ling Zhang, Xingwei Wang, Yueqin Zhang, and Fayun Guo, "Entropy-Based Global and Local Weight Adaptive Image Segmentation Models", Tsinghua Science and Technology, IEEE, vol. 25, no. DOI:10.26599/tst.2019.9010026, 2020.

[3] Mahapatra. "Semi-Supervised Learning and Graph Cuts for Consensus Based Medical Image Segmentation," Pattern Recognit., vol. 63, pp. 700-709, Mar. 2017.
[4] Mengxuan Zhang, Licheng Jiao, Ronghua Shang, Xiangrong Zhang and Lingling Li "Unsupervised EA-Based Fuzzy Clustering for Image Segmentation", IEEE Access, vol. 8, DOI: 10.1109/ACCESS.2019.2963363, 2020.

[5] Soudani, A., \&Zagrouba, E. "Adaptive Region Based Active Contour Model for Image Segmentation" IEEE/ACS 14th International Conference on Computer Systems and Applications (AICCSA), 2017.

[6] Xu, W., Yue, X., Chen, Y., \& Reformat, M. "Ensemble of Active Contour-Based Image Segmentation", IEEE International Conference on Image Processing (ICIP), 2017.

[7] Ali, H., Rada, L., \& Badshah, N. "Image Segmentation for Intensity Inhomogeneity in Presence of High Noise", IEEE Transactions on Image Processing, 27(8), pp. 3729-3738, 2018.

[8] Sasmal, P., Iwahori, Y., Bhuyan, M. K., \& Kasugai, K. "Active contour segmentation of polyps in capsule endoscopic image", 2018 International Conference on Signals and Systems (ICSigSys), 2018

[9] Pengcheng Li, Yue Zhao, Yang Liu, Qiaoyi Chen, Fangcen Liu and Chenqiang Gao "Temporally Consistent Segmentation of Brain Tissue from Longitudinal MR Data" Digital Object Identifier, IEEE Access, vol. 8, DOI: 10.1109/ACCESS.2019.2949078, 2020.

[10] Yao Yao, Changlin Xia, Jitao Li and Qiong Li, "HeadCT Image Convolution Feature Segmentation and Morphological Filtering for Densely Matching Points of IoTs", Digital Object Identifier, IEEE Access, DOI: 10.1109/ACCESS.2019.2963714, 2020.

[11] Fankui Hu, Haibing Chen and Xiaofei Wang,"An Intuitionistic Kernel-Based Fuzzy C-Means Clustering Algorithm with Local Information for Power Equipment Image Segmentation", Digital Object Identifier, IEEE Access, vol. 8, DOI: 10.1109/ACCESS.2019.2963444,2020.

[12] Yanbo Li, Junping Wang "Novel Binary Adaptive Morphological Operators", 12th IEEE International Conference on Anticounterfeiting, Security, and Identification (ASID), 2018.

[13] Amira A. Mahmoud, El-Sayed M. El-Rabaie, Taha E. Taha, Adel Elfishawy, Osama Zahran and Fathi E. Abd El-Samie, " Medical Image Segmentation Techniques, a Literature Review, and Some Novel Trends", MJEER, Vol. 27, no. 2, pp. 23-58, 2018.

[14] Soomro, S., and Choi, K. N. "Robust Active Contours for Mammogram Image Segmentation", IEEE International Conference on Image Processing (ICIP), 2017.

[15] Xiaojun Yang, Xiaoliang Jiang, Lingfei Zhou, Yong Wang and Yuliang Zhang, "Active Contours Driven by Local and Global Region-Based Information for Image Segmentation", IEEE Access, vol. 8, DOI: 10.1109/ACCESS.2019.2963435, 2020.

[16] T. Dietenbeck, M. Alessandrini, D. Friboulet, and O. Bernard, "Creaseg: A Free Software for The Evaluation of Image Segmentation Algorithms Based on Level-Set", in IEEE International Conference on Image Processing. Hong Kong, China, 2010.

[17] http://www.onlinemedicalimages.com/index.php/en/

[18] https://www.cancerimagingarchive.net/

[19] Dang N. H. Thanh "Image Segmentation Quality Scores", (https://www.mathworks.com/matlabcentral/fileexchange/71221, (image segmentation quality-scores), MATLAB Central File Exchange,

2019. 